\title{
Effects of green tea epigallocatechin-3-gallate on the proteolipid protein and oligodendrocyte transcription factor 1 messenger RNA gene expression in a mouse model of multiple sclerosis
}

\author{
Mohammadreza Semnani ${ }^{1}$, Farhad Mashayekhi ${ }^{2}$, Mahnaz Azarnia ${ }^{1}$, Zivar Salehi ${ }^{2}$ \\ ${ }^{1}$ Kharazmi University, Tehran, ${ }^{2}$ University of Guilan, Rasht, Guilan, Iran
}

\begin{abstract}
The cuprizone multiple sclerosis (MS) animal model is characteristic for toxic demyelination and represents a reversible demyelination and remyelination system. It has been shown that green tea epigallocatechin-3-gallate (EGCG) might be effective in improving the symptoms and pathological conditions associated with autoimmune inflammatory diseases in several animal models. In this study the effects of EGCG on proteolipid protein (PLP) and oligodendrocyte transcription factor 1 (Olig1) expression in the cerebral cortex of a murine model of cuprizone-induced demyelination was investigated. C57BL/6 mice were treated with cuprizone for six weeks in order to induce demyelination. Immediately after the cessation of cuprizone the animals were divided into 6 groups ( $n=10$ for each group). The first two groups were injected intraperitoneally (IP) with EGCG in the amount of $50 \mathrm{mg} / \mathrm{kg} /$ daily body weight for 2 and 4 weeks. The second two groups (SHAM) were injected IP with phosphate-buffered saline (PBS) for 2 and 4 weeks, and the third two groups were left without injection as controls. After two and four weeks the mice were killed and the cerebral cortex was collected and the expression of Plp and Olig1 was studied by real-time PCR. The results showed significant increases in PLP and Olig1 expression in the EGCG-treated groups as compared to the SHAM and control groups $(p<0.0001)$. It is concluded that EGCG increases PLP and Olig1 expression in the cerebral cortex of a mouse model of MS induced by cuprizone.
\end{abstract}

Key words: cuprizone, EAE, EGCG, Plp, Olig1.

\section{Introduction}

Multiple sclerosis (MS) is the most common cause of non-traumatic disabilities among young people, affecting approximately two and a half million people from around the world [20]. The etiology of MS is not known yet and it may include some genetic susceptibilities, epigenetic regulations and environmental factors [4]. It has been shown that genetics and environmental factors are involved in the pathology of MS. Accumulating evidence indicates that oxidative stress is an important factor involved in the pathogenesis of MS. Oxidative stress activates some mechanisms that lead to glia-mediated inflammation and neural damage [16].

The cuprizone model is characteristic for toxic demyelination and it represents reversible demyelin- 
ation and remyelination. Cuprizone induces reversible demyelination in several brain regions including the hippocampus, cerebellum, caudate putamen, corpus callosum and grey matter regions. It is still not clear whether the copper chelating nature of cuprizone is implicated in its neurotoxic properties [15]. Additional mechanisms such as inhibition of oligodendrocyte progenitor cell (OPC) differentiation [3] and secretion of pro-inflammatory cytokines from activated microglia/macrophages are also discussed [24].

The multilayer central nervous system (CNS) myelin membrane sheaths that surround the nerve fibers (axons) are formed by lipids and proteins distributed according to the charge, lipo- or hydrophilicity, and relative molecular weight [27]. In the CNS the main proteins of myelin are proteolipid protein (PLP), myelin basic protein (MBP), myelin oligodendrocyte glycoprotein (MOG) and CNPase. Myelin oligodendrocyte glycoprotein is an important autoantigen linked to the pathogenesis of multiple sclerosis [21].

In the CNS, PLP and its alternative spliced isoform, DM20, make up the bulk of the proteolipids [10]. Proteolipid protein and DM20 constitute more than 50\% of the total protein of CNS myelin. PLP1, the gene encoding PLP and DM20, is located on the X chromosome. Multiple copies of $P L P 1$, point mutations or deletion of $P L P 1$ can lead to X-linked dysmyelinating disorders, Pelizaeus-Merzbacher disease, and spastic paraplegia type $2[6,34]$. It has also been suggested that PLP interacts with integrin receptors in order to affect signal transduction in oligodendrocytes and homing of immature oligodendrocytes. Clearly, PLP has an important role in supporting normal axonal functions. It is believed that PLP and DM20 are likely the major targets of the autoimmune responses in MS, since they are highly encephalitogenic in animals and auto-reactivity directed against PLP is elevated in patients with MS [10].

Oligodendrocyte transcription factor 1 (Olig1), a stage-specific marker for oligodendrocytes in the brain, plays a key role in the regulation of the myelin-specific gene expression and the program of myelinogenesis. Olig1 plays a pivotal role in oligodendrocyte myelination. Ablation of Olig1 leads to the absence of all major myelin specific gene expression inside the brain and complete failure of myelin sheath formation. Moreover, Olig1-null mice develop prominent axonal degeneration, swellings, and gliosis in the CNS [35].

Genetics and environmental factors including nutrition were shown to be important in the remyelination process. Polyphenols are natural phytochemicals and they are secondary metabolites produced in plants to defend themselves from cellular stress. The interest in polyphenols is related to their well-known antioxidant activities which are reported to have positive effects on human health, due to their presence in dietary sources such as fruits, vegetables, wine, spices and herbs [16]. Green tea, prepared from the leaves of Camellia sinensis, is one of the popular beverages that are reported to have a number of beneficial health effects, including anti-inflammatory, anti-thrombotic and anticancer activities [12]. Green tea is rich in polyphenolic compounds, known as catechins, that are responsible for the physiological activity of green tea and its extracts. One of the main catechins found in green tea is epigallocatechin-3-gallate (EGCG) [2]. Accumulating evidence has revealed an immunomodulating effect of EGCG. Several types of immune cells in both the innate and adaptive immune systems are known to be affected by EGCG [23]. Epigallocatechin-3-gallate is capable of protecting against neuronal injury in brain tissue induced by $N$-methyl- $D$-aspartate and blocking the formation of neurotoxic reactive oxygen species in neurons [1]. Epigallocatechin-3-gallate attenuated symptoms and pathological features (leukocyte infiltration and demyelination) in the CNS and inhibited antigen specific T-cell proliferation and delayed type hypersensitivity of skin responses. These results demonstrate that EGCG may attenuate experimental autoimmune encephalomyelitis (EAE) autoimmune responses by inhibiting immune cell infiltration and modulating the balance among pro- and anti-autoimmune $\mathrm{CD}^{+}{ }^{+}$T-cells [33]. It was shown that EGCG might be the most promising neuroprotective compound. Thus, inclusion of this polyphenol in the diet might provide an affordable means to reduce the impact of neurodegenerative diseases [25].

Most studies on the effects of EGCG on autoimmune disease including MS have concentrated on its anti-inflammatory properties. In this study, for the first time, we aimed to investigate the effects of EGCG in green tea on the expression of PLP and Olig1 in cuprizone-induced demyelinated mice using real-time $P C R$. 


\section{Material and methods Animals}

C57BL/6 mice were purchased from Pasteur Institute, Tehran, Iran and maintained on a 12-12 light: dark cycle beginning at $8.00 \mathrm{am}$. They were kept at a constant temperature in mouse boxes with unrestricted access to laboratory food and water. The colony was maintained through random pair mating. Cage maintenance was performed once a week and the animals were handled by the same individuals throughout the experimental period. Food and tap water were available ad libitum throughout the acclimatization and experimental period. All animal procedures were carried out in accordance with the Animals (Scientific Procedure) Act, 1986. All animal protocols used have been approved by the authors' institutional animal experimentation committee. Sixty male C57BL/6 mice aged 8 weeks were included in this study.

\section{Induction of demyelination}

Demyelination was induced by feeding 8-weekold mice a diet containing $0.2 \%$ cuprizone (biscyclohexanone oxaldihydrazone, Sigma-Aldrich Inc.) mixed into ground standard rodent chow. The cuprizone diet was administered for 6 weeks for demyelination. In order to evaluate MS induction by cuprizone, animals were tested on a rotarod to measure changes in balance and motor coordination.

\section{Motor co-ordination and learning: rotarod test}

Motor co-ordination and balance was evaluated in a rotarod apparatus (Letica Scientific Instruments) which consisted of a motor-driven rotating rod equipped with variable speeds. All mice were evaluated on the rotarod three times a day (in the afternoon) for three consecutive days with the rotation set at 16, 24 or 32 revolutions per minute (rpm) [9].

\section{Epigallocatechin-3-gallate administration}

Immediately after the cessation of cuprizone the animals were divided into 6 groups $(n=10$ for each group). The first two groups was injected intraperitoneally (IP) with EGCG in the amount of $50 \mathrm{mg} / \mathrm{kg} /$ daily body weight for 2 and 4 weeks. The second two groups (SHAM) were injected i.p. with phosphate-buffered saline (PBS) for 2 and 4 weeks, and the third two groups were left without injection as controls. After two and four weeks the cerebral cortex was collected after euthanasia by intraperitoneal injection of an overdose of anesthetic (sodium pentobarbitone) and processed as described. A total of 60 animals were used in this study.

\section{RNA extraction and cDNA preparation}

For real-time PCR analysis, RNA was isolated from the cerebral cortex using a TRIzol kit. To produce cDNA, equal amounts of RNA were reverse-transcribed in a standard reaction with reverse transcriptase.

\section{Real-time PCR}

In order to perform the real-time PCR for Plp and Olig1 genes, primers were designed using Oligo 7 software. The reference gene for this method was GAPDH and it was amplified using the provided forward and reverse primers of the first strand cDNA synthesis kit. Forward and reverse primer sequences and lengths of Plp and Olig1 genes are shown in Table I. Thermal profiles of Plp, Olig1 and GAPDH were identical as follows: The reaction was initiated at $94^{\circ} \mathrm{C}$ for 3 minutes followed by 39 continuous cycles of $94^{\circ} \mathrm{C}$ for 30 seconds, $58^{\circ} \mathrm{C}$ for 30 seconds and $72^{\circ} \mathrm{C}$ for 45 seconds. The last stage of the reaction was performed at $65^{\circ} \mathrm{C}$ for 5 seconds.

\section{Statistical analysis}

The quantity of gene expression was analyzed as described by Livak and Schmittgen [17]. Statistical analysis was performed using IBM SPSS Statistics (Version 21), with one-way analysis of variance (ANOVA) followed by Tukey's post-hoc test. A probability of $p<0.05$ was considered statistically significant. Graphs were made using Excel.

Table I. Forward and reverse primer sequences and lengths of Plp and Olig1

\begin{tabular}{|llc|}
\hline Primer & \multicolumn{1}{c|}{ Sequences } & Length (nt) \\
\hline Forward Plp & TGAGCAAAGTCAGCCGCAAA & 20 \\
\hline Reverse Plp & CCCCTACCAGACATCTAGCAC & 21 \\
\hline Forward Olig1 & GCCCCACCAAGTACCTGTC & 19 \\
\hline Reverse Olig1 & GGAACTTGCACACCGCACA & 19 \\
\hline
\end{tabular}




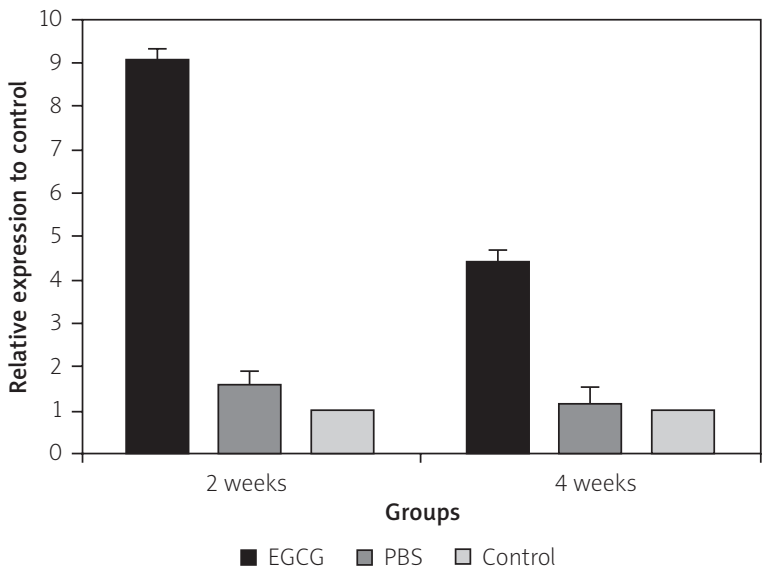

Fig. 1. Plp expression in response to treatment with epigallocatechin-3-gallate (EGCG). Both of the studied groups ( 2 and 4 weeks) showed a significant increase in Plp gene expression in the EGCG-treated group as compared to SHAM and control groups $(p<0.0001)$ (mean \pm SE).

\section{Results}

\section{Motor co-ordination and learning: rotarod test}

Motor co-ordination was evaluated using a rotarod apparatus. All animals improved their skill and learned to stay on the progressively speeded-up rotating rod. Rota-rod analysis demonstrated that the treated group had poorer motor co-ordination than control animals $(p<0.05)$.

\section{Real-time PCR}

Products of the real-time PCR reaction were subjected to $2 \%$ agarose gel electrophoresis containing ethidium bromide and the proliferated fragments were observed using UV. The length of the targeted area within Plp, Olig1 and GAPDH was 105, 114 and $495 \mathrm{bp}$, respectively.

The results showed that Plp expression in 2 and 4 weeks treated EGCG groups was increased as compared to SHAM and control groups. Relative expression of Plp in the cases of 2 weeks of treatment with EGCG and SHAM was 9.06 and 1.63, respectively, as compared to the control group (with the expression of 1). Moreover, relative expression of Plp in the cases of 4 weeks of treatment and SHAM was 4.42 and 1.15 , respectively, as compared to the control group (with the expression of 1) (Fig. 1). Statistical analy-

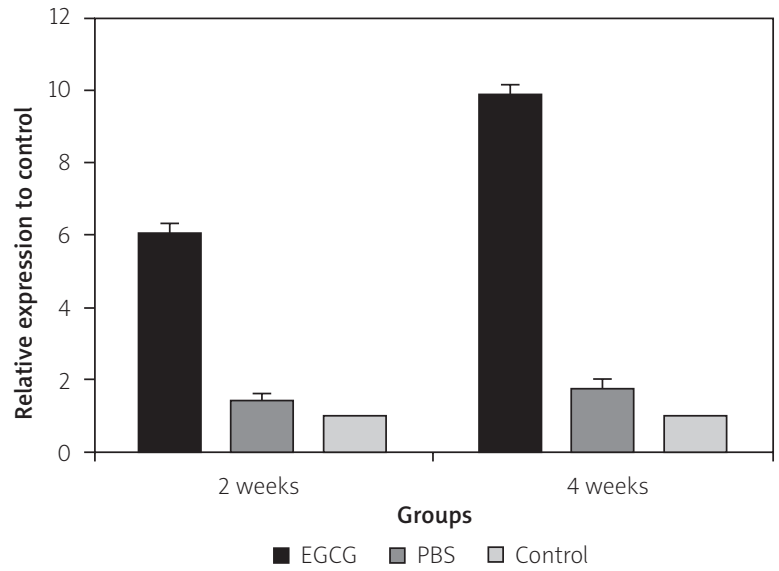

Fig. 2. Olig1 expression in response to treatment with epigallocatechin-3-gallate (EGCG). Both of the studied groups (2 and 4 weeks) showed a significant increase in Olig1 gene expression in the EGCG-treated group as compared to SHAM and control groups $(p<0.0001)$ (mean $\pm \mathrm{SE}$ ).

sis showed a significant increase in the expression of Plp in the 2 and 4 weeks treated groups as compared to either SHAM or controls ( $p<0.0001)$.

The result of real-time PCR in the case of the Olig1 gene indicated an increase in gene expression among 2 and 4 weeks EGCG-treated groups as compared to the SHAM and controls. Relative increase of Olig1 expression among the 2 weeks treated group and SHAM compared to the control group (with the expression of 1) was 6.11 and 1.39 , respectively. Relative expression of Olig1 in the cases of 4 weeks of treatment with EGCG and SHAM was 9.98 and 1.71 , respectively, as compared to the control group (with the expression of 1) (Fig. 2). Statistical analysis showed a significant increase in the expression of Olig1 in the 2 and 4 weeks treated groups ( $p<0.0001)$. We found no significant change in Plp and Olig1 expression between SHAM and controls.

\section{Discussion}

Multiple sclerosis is one of the T-cell-mediated inflammatory autoimmune diseases of the CNS. Experimental autoimmune encephalomyelitis is a wellestablished animal model for human MS, owing to the similarities in clinical, neuropathological, and immunological features between MS and EAE [8]. Both genetic background and environmental factors 
are involved in the pathogenesis of MS. The current therapies that mainly focus on the application of immune suppressant drugs have limited efficacy and various adverse effects. Nutrition represents a secondary and complementary approach that could potentially improve the autoimmune disorders [36]. It includes the effects of certain vitamins and food components in the pathology and treatment of MS. Recent studies suggest that vitamin $B_{12}$, in addition to its known role as a co-factor in myelin formation, has important immunomodulatory and neurotrophic effects [19]. It has been shown that low vitamin D intake or serum vitamin D status is associated with increased risk of developing MS [22]. It has been documented that EGCG given to patients with MS over 12 weeks improves muscle metabolism during moderate exercise to a greater extent in men than in women, possibly because of sex-specific effects on autonomic and endocrine control [18].

Dietary EGCG supplementation is helpful in ameliorating the symptoms and pathological changes in EAE animals [30]. The effect of EGCG is associated with reduced inflammatory infiltration in the CNS and reduced proliferation of auto-reactive T-cells and their differentiation into different subsets with corresponding responsive functions. Administration of EGCG to EAE mice reduced brain inflammation, clinical symptoms, and neuronal damage. It was shown that treatment with EGCG reduced plasma levels of soluble intercellular adhesion molecule- 1 and $\mathrm{CD}^{+}{ }^{+}$T-cells expressing C-C chemokine receptor 6 (CCR6) in EAE mice [33]. It was also shown that EGCG treatment induced up-regulation of anti-apoptotic Bcl-2 and down-regulation of proapoptotic Bax in injured spinal tissues [14]. Epigallocatechin-3-gallate treatments significantly attenuated the expression of IL- $1 \beta$ and TNF- $\alpha$, and consequently expression of iNOS and COX-2 [31]. Besides their anti-oxidative and anti-inflammatory properties, many phenolics may also have specific action on intracellular signaling pathways. Green tea extract may also exert protection through controlling calcium homeostasis, activation of MAPK, PKC, antioxidant enzymes, survival genes and modulating enzymes for processing of the amyloid precursor protein [29]. It has been shown that while EGCG induced down-regulation of the gene expression of heme oxygenase-1 (HO-1) in affected CNS areas, the combined therapy of glatiramer acetate (GA) and EGCG seems to promote increased HO-1 expression. These data suggest that up-regulation of HO-1 may contribute to diminished neuroprotective benefits of EGCG alone in this EAE model [11]. It has been shown that usage of preparations containing pig spinal cord hydrolysate, as a source of broad spectrum myelin antigens, is able to diminish clinical symptoms of ongoing EAE [13].

Green tea consumption improves both reflexes and sensation in unilateral chronic constriction injury to the sciatic nerve. Histological examination of sciatic nerves from EGCG-treated rats showed that the axotomized animals had remarkable axonal and myelin regeneration with a significant decrease in the number of myelinated axonal fibers compared to the vehicle-treated crush group. Epigallocatechin-3-gallate-treated rats showed a significant increase in paw withdrawal thresholds to mechanical stimulation compared to the vehicle-treated crush group. Epigallocatechin-3-gallate treatment also restored the mRNA expression of Bax, Bcl-2 and survivin but not that of p53 to SHAM levels on post-injury days 3 and 7. Overall, EGCG treatment enhanced functional recovery, advanced morphological nerve rescue and accelerated nerve regeneration following crush injury partly due to the down-regulation of apoptosis-related genes [26].

It has been shown that resveratrol, a type of natural phenol, effectively enhanced motor coordination and balance, reversed cuprizone-induced demyelination, improved mitochondrial function, alleviated oxidative stress, and inhibited NF- $\mathrm{KB}$ signaling. Interestingly, resveratrol increased Olig1 expression, which is positively correlated with active remyelination. These data also suggest that resveratrol promoted oligodendrocyte progenitor cell differentiation and consequently remyelination as evidenced by improved balance and motor coordination in cuprizone-intoxicated animals [7].

It was reported that EGCG triggered a cascade of events including activation of phospholipase C, mobilization of intracellular calcium stores, a rise of cytosolic $\mathrm{Ca}^{2+}$ levels, and inhibition of VGCC and NMDA ( $N$-methyl- $D$-aspartate) receptor-mediated $\mathrm{Ca}^{2+}$ influxes [32]. It has also been shown that glutamate receptor antagonists have a positive effect on the expression of mRNA MBP and glycoproteins MAG and MOG [5].

Using western blotting, it was shown that EGCG increases proteolipid protein (PLP) and oligodendrocyte transcription factor 1 (Olig1) expression at 
the protein level in the cerebral cortex of cuprizoneinduced multiple sclerosis mice [28]. These results strongly suggest a promising potential for using EGCG as a therapeutic agent in MS and possibly in other T-cell mediated autoimmune diseases [33].

In this study we have shown for the first time that EGCG increases Plp and Olig1 expression in the cerebral cortex of cuprizone-induced MS mice. Since PLP is one of the major components of the myelin sheath, an increase of its expression in response to EGCG in cuprizone-treated mice may be involved in the remyelination process. Furthermore, as OLIG1 is essential for the process of proliferation and differentiation of oligodendrocyte progenitor cells, increased expression of Olig1 may be important in the remyelination process.

We also found that Olig1 expression was higher in the 4-week treatment as compared to the 2-week treatment. It seems that since Olig1 has an important role in the late stages of oligodendrocyte differentiation and correction of the remyelination process, the continuation of treatment with EGCG is more effective due to the special role of Olig1.

It is concluded that EGCG increases PLP and Olig1 expression in the cerebral cortex of a mouse model of MS induced by cuprizone.

\section{Acknowledgements}

We would like to thank the Department of Cell and Molecular Biology, School of Biological Sciences, Kharazmi University, and the Department of Biology, Faculty of Sciences, University of Guilan for technical assistance.

\section{Disclosure}

Authors report no conflict of interest.

\section{References}

1. Aktas O, Prozorovski T, Smorodchenko A, Savaskan NE, Lauster R, Kloetzelm PM, InfanteDuarte C, Brocke S, Zipp F. Green tea epigallocatechin-3-gallate mediates T cellular NF-kappa B inhibition and exerts neuroprotection in autoimmune encephalomyelitis. J Immunol 2004; 173: 5794-5800.

2. Balentine DA, Wiseman SA, Bouwens LC. The chemistry of tea flavonoids. Food Sci Nutr 1997; 37: 693-704.

3. Cammer W. The neurotoxicant, cuprizone, retards the differentiation of oligodendrocytes in vitro. J Neurol Sci 1999; 168: 116-120.

4. Costenbader KH, Gay S, Alarcón-Riquelme ME, laccarino L, Doria A. Genes, epigenetic regulation and environmental fac- tors: which is the most relevant in developing autoimmune diseases? Autoimmun Rev 2012; 11: 604-609.

5. Dąbrowska-Bouta B, Strużyńska L, Chalimoniuk M, FrontczakBaniewicz M, Sulkowski G. The influence of glutamatergic receptor antagonists on biochemical and ultrastructural changes in myelin membranes of rats subjected to experimental autoimmune encephalomyelitis. Folia Neuropathol 2015; 53: 317-326.

6. Garbern JY. Pelizaeus-Merzbacher disease: Genetic and cellular pathogenesis. Cell Mol Life Sci 2007; 64: 50-65.

7. Ghaiad HR, Nooh MM, El-Sawalhi MM, Shaheen AA. Resveratrol Promotes Remyelination in Cuprizone Model of Multiple Sclerosis: Biochemical and Histological Study. Mol Neurobiol 2017; 54: 3219-3229.

8. Gold R, Hartung HP, Toyka KV. Animal models for autoimmune demyelinating disorders of the nervous system. Mol Med Today 2000; 6: 88-91.

9. Golub MS, Germann SL, Lloyd KC. Behavioral characteristics of a nervous system-specific erbB4 knock-out mouse. Behav Brain Res 2004; 153: 159-170.

10. Greer JM, Lees MB. Myelin proteolipid protein - the first 50 years. Int J Biochem Cell Biol 2002; 34: 211-215.

11. Janssen A, Fiebiger S, Bros H, Hertwig L, Romero-Suarez S, Hamann I, Chanvillard C, Bellmann-Strobl J, Paul F, Millward JM, Infante-Duarte C. Treatment of Chronic Experimental Autoimmune Encephalomyelitis with Epigallocatechin-3-Gallate and Glatiramer Acetate Alters Expression of Heme-Oxygenase-1. PLoS One 2015; 10: e0130251.

12. Kang J, Cheng H, Ji J, Incardona J, Rampe D. In vitro electrocardiographic and cardiac ion channel effects of (-)-epigallocatechin-3-gallate, the main catechin of green tea. J Pharmacol Exp Ther 2010; 334: 619-626.

13. Kasarełło K, Gadamski R, Piotrowski P, Kurzepa K, Kwiatkowska-Patzer B, Lipkowski AW. Effect of oral administration of pig spinal cord hydrolysate on clinical and histopathological symptoms of experimental allergic encephalomyelitis in rats. Folia Neuropathol 2015; 53: 128-138.

14. Khalatbary AR, Tiraihi T, Boroujeni MB, Ahmadvand H, Tavafi M, Tamjidipoor A. Effects of epigallocatechin gallate on tissue protection and functional recovery after contusive spinal cord injury in rats. Brain Res 2010; 1306: 168-175.

15. Kipp M, Clarner T, Dang J, Copray S, Beyer C. The cuprizone animal model: new insights into an old story. Acta Neuropathol 2009; 118: 723-736.

16. Liuzzi GM, Latronico T, Branà MT, Gramegna P, Coniglio MG, Rossano R, Larocca M, Riccio P. Structure-dependent inhibition of gelatinases by dietary antioxidants in rat astrocytes and sera of multiple sclerosis patients. Neurochem Res 2011; 36 : 518-527.

17. Livak KJ, Schmittgen TD. Analysis of relative gene expression data using real-time quantitative PCR and the 2(-Delta Delta C(T)) Method. Methods 2001; 25: 402-408.

18. Mähler A, Steiniger J, Bock M, Klug L, Parreidt N, Lorenz M, Zimmermann BF, Krannich A, Paul F, Boschmann M. Metabolic response to epigallocatechin-3-gallate in relapsing-remitting multiple sclerosis: a randomized clinical trial. Am J Clin Nutr 2015; 101: 487-495. 
19. Miller A, Korem M, Almog R, Galboiz Y. Vitamin B12, demyelination, remyelination and repair in multiple sclerosis. J Neurol Sci 2005; 233: 93-97.

20. Münzel EJ, Williams A. Promoting remyelination in multiple sclerosis-recent advances. Drugs 2013; 73: 2017-2029.

21. Norton WT, Autilio LA. The lipid composition of purified bovine brain myelin. J Neurochem 1966; 13: 213-222.

22. O'Gorman C, Lucas R, Taylor B. Environmental risk factors for multiple sclerosis: a review with a focus on molecular mechanisms. Int J Mol Sci 2012; 13: 11718-11752.

23. Pae M, Wu D. Immunomodulating effects of epigallocatechin-3-gallate from green tea: mechanisms and applications. Food Funct 2013; 4: 1287-1303.

24. Pasquini LA, Calatayud CA, Bertone Uña AL, Millet V, Pasquini JM, Soto EF. The neurotoxic effect of cuprizone on oligodendrocytes depends on the presence of pro-inflammatory cytokines secreted by microglia. Neurochem Res 2007; 32: 279-292.

25. Pogačnik L, Pirc K, Palmela I, Skrt M, Kim KS, Brites D, Brito MA, Ulrih NP, Silva RF. Potential for brain accessibility and analysis of stability of selected flavonoids in relation to neuroprotection in vitro. Brain Res 2016; 1651: 17-26.

26. Renno WM, Al-Maghrebi M, Alshammari A, George P. (-)-Epigallocatechin-3-gallate (EGCG) attenuates peripheral nerve degeneration in rat sciatic nerve crush injury. Neurochem Int 2013; 62: 221-231.

27. Rosetti CM, Maggio B, Oliveira RG. The self organization of lipids and proteins of myelin at the membrane interface. Molecular factors underlying the microheterogeneity of domain segregation. Biochim Biophys Acta 2008; 1778: 1665-1675.

28. Semnani M, Mashayekhi F, Azarnia M, Salehi Z. Effects of Green Tea Epigallocatechin-3-Gallate (EGCG) On Proteolipid Protein (PLP) and Oligodendrocyte Transcription Factor 1 (Olig1) Expression in the Cerebral Cortex of Cuprizone Induced Multiple Sclerosis Mice; A Western Blot Study. Caspian I Neurol Sci 2016; 2: 1-9.

29. Sun AY, Wang Q, Simonyi A, Sun GY. Botanical phenolics and brain health. Neuromolecular Med 2008; 10: 259-274.

30. Sun O, Zheng Y, Zhang X, Hu X, Wang Y, Zhang S, Zhang D, Nie H. Novel immunoregulatory properties of EGCG on reducing inflammation in EAE. Front Biosci (Landmark Ed) 2013; 18: 332-342.

31. Sutherland BA, Rahman RM, Appleton I. Mechanisms of action of green tea catechins, with a focus on ischemia-induced neurodegeneration. J Nutr Biochem 2006; 17: 291-306.

32. Wang J, Cheng J, Li CR, Ye M, Ma Z, Cai F. Modulation of $\mathrm{Ca}^{2+}$ signals by epigallocatechin-3-gallate(EGCG) in cultured rat hippocampal neurons. Int I Mol Sci 2011; 12: 742-754.

33. Wang J, Ren Z, Xu Y, Xiao S, Meydani SN, Wu D. Epigallocatechin-3-gallate ameliorates experimental autoimmune encephalomyelitis by altering balance among CD4+ T-cell subsets. Am J Pathol 2012; 180: 221-234.

34. Woodward KJ. The molecular and cellular defects underlying Pelizaeus-Merzbacher disease. Expert Rev Mol Med 2008; 10: e14.

35. Xin M, Yue T, Ma Z, Wu FF, Gow A, Lu QR. Myelinogenesis and axonal recognition by oligodendrocytes in brain are uncoupled in Olig1-null mice. J Neurosci 2005; 25: 1354-1365.

36. Yang CS, Maliakal P, Meng X. Inhibition of carcinogenesis by tea. Annu Rev Pharmacol Toxicol 2002; 42: 25-54. 